\title{
The Use of Digital Photo Frames as Situated Messaging Appliances
}

\author{
John Downs, Bery1 Plimmer \\ Department of Computer Science, University of Auckland, New Zealand \\ jdow038@ec.auckland.ac.nz, beryl@cs.auckland.ac.nz
}

\begin{abstract}
SMS messaging has traditionally been utilised for person-to-person messaging. Some researchers have recognised the value in using SMS for person-to-location messaging, but such technology has been largely confined to the laboratory. We argue that commercially available digital photo frames provide a low-cost opportunity for situated display technology, and that such technology can be used for person-to-location SMS messaging. This paper describes an initial prototype of the software infrastructure that provides this functionality, our plans for its evaluation and suggests possible extensions and improvements for future consideration.
\end{abstract}

Keywords: SMS messaging; social computing; digital photo frames; situated displays; information appliances

\section{Introduction}

SMS messaging is an extremely popular messaging technology, used extensively for person-to-pers on communication. The success of the technology is largely based on the low cost of sending and receiving messages, the technology's relative ease of use, and the asynchronous nature of SMS messages: messages are sent and delivered quickly, but are picked up at a future time of the recip ient's choosing. As noted in [1], there are also situations where it is advantageous to send such asynchronous messages to specific locations, such as one's home or office, rather than to an individual person. As mobile phones gradually reduce households' reliance on landlines, location-based text messaging provides a replacement person-to-location messaging technology that allows for the best of both phone-based and location-based messaging. However, phone-based SMS messaging does not currently include the capability for this type of messaging.

Digital photo frames are becoming cheaper and more feature-rich, but are still almost exclusively focused on retrieving, displaying, and working with digital photographs. Newer frames are capable of connecting to wireless networks and retrieving photographs fro m co mputers without the use of memory cards, USB cables, or USB flash memory 'sticks'. Considering they are essentially miniature computer displays, it seems that their capability to display information beyond simple photographs is underutilised. This project examines how a digital photo frame might 
be used for purposes beyond those for which it was originally designed-namely, SMS messaging.

\section{Background}

Studies of situated display technology have traditionally focused on improving efficiency and communication opportunities in offices and other workplaces by placing dedicated, special-purpose computer displays in everyday working environments. Example studies include displays to show information relating to meeting room reservations [2], and office door plates [3]. This technology has become so readily available and cost-effective that it is now commonplace-for example, the University of Auckland displays lecture theatre booking information on a display situated outside lecture rooms, and similar displays are used to show the hours of operation for undergraduate computer laboratories. Researchers have also started to study the impact that this type of technology can have in the home. Studies of the design and creation of a digital family calendar for family co-ordination [4], an interactive appliance used to send and receive SMS messages [5], and a location awareness appliance that displays information about family members' locations [6] are examp les of this trend. Based on research by Beech et al. [7] one can argue that all of these appliances serve a useful and practical purpose. These appliances can also be considered a part of the paradigm of 'social computing'-the use of computer technology to enable, support, and enhance interpersonal communication.

The majority of research that has been conducted on home-based situated awareness appliances has focused on creating interactive devices that are suited for a single purpose, often by adapting technology such as laptops or tablet PCs, or by creating and using specialised technology. While proving useful for researchers, this type of research is limited in its real-world applications, as it does not provide an easy path for users to begin to embed this technology into their everyday lives. Accordingly, relatively little of this technology has actually been commercialised. Digital photo frames, however, are increasingly being used by families to display digital photos, and therefore beginning to embed digital information into their home lives. Despite their prevalence, little research has been conducted on using these photo frames for purposes beyond displaying photographs. This study attempts to examine the extent to which digital photo frames can be used for one specific function-unidirectional SMS messaging-and, more broadly, the ways in which digital photo frames might be able to be used as cost-effective and highly available information awareness appliances.

\section{Digital Photo Frame Management System}

We expect that digital photo frames will provide a relatively low-cost platform on which to create non-interactive applications for situated displays, and that this research will help to define the parameters for which these types of applications are useful. Additionally, and equally important, we expect that households will find the 
ability to send SMS messages to a specific place in the home to be a useful application of this technology.

\subsection{Software}

Custom software is required in order to display the messages on the photo frames. This software is composed of two primary components (Fig. 1), each implemented as a Windows service, using Microsoft Visual Studio 2008 and running on the Microsoft .NET Framework version 3.5, and a shared Microsoft SQL Server 2005 database.

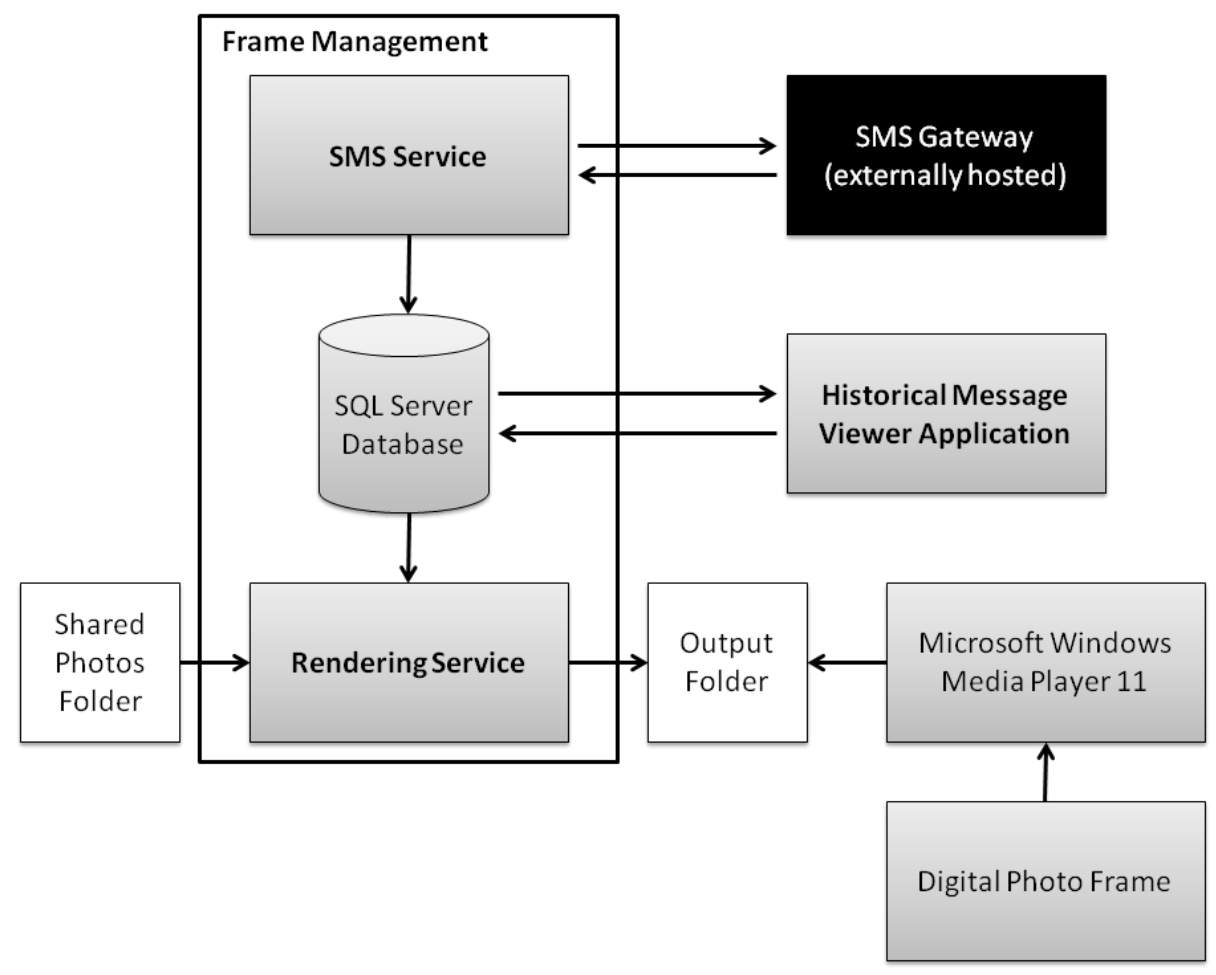

Fig. 1. Components of the digital photo frame management system.

The first Windows service, the SMS service, periodically polls the SMS gateway service for new messages for the frame. The service can reject messages that are not sent from a recognised mobile number. By default this polling occurs every 30 seconds. When new messages arrive, the SMS service records them into the database (hosted on the same machine).

The second service, the rendering service, receives notifications from the database when a new SMS message has been received, and then begins the rendering process. Since photo frames work exclusively with images (usually in JPEG format), the software is required to render messages into JPEG format and then send them to the frame to be displayed. Communication with the frame uses a standard protocol; the 
Kodak EASYSHARE Ex1011 frames we use communicate using the UPnP AV Media Server protocol [8]. Client applications such as Microsoft Windows Media Player 11 provide the user interface to manage the images. Our frame management software uses Windows Media Player's UPnP media server to send the images to the photo frame.

The computer running the software hosts a shared network folder into which users can place digital photos they wish to have displayed on the frame. The rendering process consists of enumerating the digital photos that the user wishes to display and modifying each of these photos to include the messaging information in a panel on the right side of the frame, obscuring approximately one third of the photo (Fig. 2). If no messages are present the message panel is not displayed.

As the images are updated, the rendering service places them into a folder that Windows Media Player monitors, and removes any images from previous rendering iterations. When Windows Media Player detects the change in files it notifies the digital photo frame through the UPnP AV protocol, and the new images are shown on the frame's display. Communication between the frame and the computer occurs wirelessly in order to allow maximum flexibility when positioning the frame. Network connections between other system components may be wired or wireless depending on the network configuration.

Frame. Due to the non-interactivity of digital photo frames, there is no capability for annotating or deleting messages. Instead, as new messages come in, existing messages gradually exit the frame. Depending on the length of each message, several messages are displayed on the frame at any given time. Historical messages that are no longer on the frame are accessible through a Windows application on the host PC. This application lists the complete history of messages that have been received by the system.

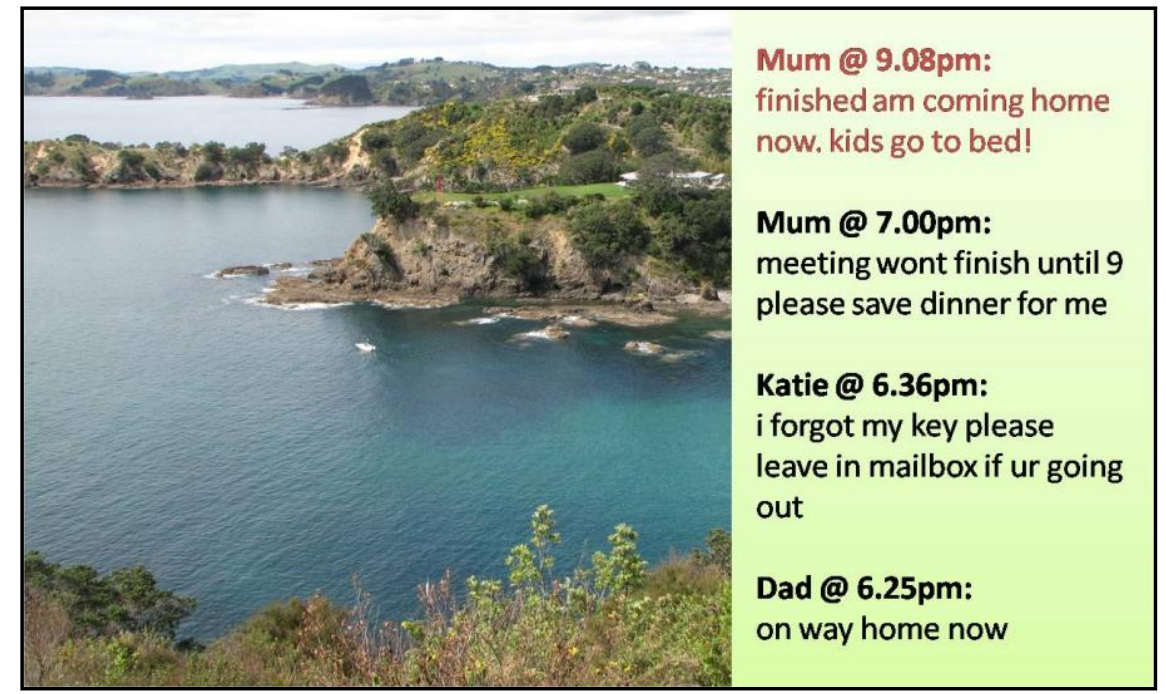

Fig. 2. Example of an image after messages have been received and rendered. 


\section{Future Work}

This technology has been developed and preliminary testing of the software has been completed. Planning is underway for a more comprehensive evaluation study of the system as a whole, which will indicate the extent to which this type of photo frame technology can be used in practical situations.

\subsection{Ethnographic Evaluation Study}

Dig ital photo frames and computers running the appropriate software will be provided to households participating in an ethnographic evaluation study. To get an overall impression of the usefulness of the technology, and to compare usage patterns, we plan for two households to participate in the pilot study. One household will consist of a family (parents and children), at least some of whom use SMS to communicate with each other on a regular basis. The other household will consist of a flat of at least three young adults who also regularly communicate with each other using SMS.

When the evaluation study begins, members of these households will be asked to participate in an interview to discuss their SMS usage and current methods of communicating with the household. The study will take place over approximately one month for each household. Once during the study, and again at the end of the study, the household will be interviewed for their impressions of the technology and to determine whether they find it (and the communication it affords) to be useful. Similar to [5], a log of the SMS messages the household sent will be grouped and categorised to determine what types of messaging the household found most us eful.

\subsection{Extensions}

The software itself can be extended and enhanced in a number of ways. It would be possible (and desirable) for the system to host its own UPnP AV server rather than using Windows Media Player. In the present implementation, the only pictures that can be stored in the Windows Media Player library are the output images from the rendering service. Hosting a UPnP server directly would eliminate this constraint and would enable digital photos to be dynamically modified with content when requested by the frame, rather than requiring the software to prepare the images in advance.

Additionally, there is wide scope for the provision of additional content, instead of or in addition to SMS messages. News, weather information, RSS feeds, family status updates, and other types of content may all be useful candidates for information for non-interactive household situated displays. By extending and enhancing the software described here, future studies can easily and cost-effectively implement and test these applications.

This project only examines the use of static images. Digital photo frames are now capable of displaying video, even across a network, and can play audio. This has implications for the usefulness of our technology-it may be advantageous, for 
example, to play an audio alert when a new SMS message arrives. Additionally, if the software rendered video rather than static images, it may be possible to enable additional effects such as highlighting of new messages in order to capture attention, the scrolling of messages (which would also allow for an increased number of messages to be displayed), and animation effects to improve aesthetics. Finally, it may be possible to integrate the messaging system with MMS (phone-based multimedia messaging) and to allow users to send photos, videos, and audio to the frame. From our pilot study we hope to gauge the level of interest in these other content types and features.

\section{Conclusion}

By coupling SMS messaging technology with commercially available digital photo frames we have created a low-cost situated display for the home. The technology provides the opportunity for future extensibility of content types and of output format, allowing for its adaptation to general-purpose situated displays. The next phase of this project is to conduct an ethnographic evaluation study to evaluate the usefulness of this type of technology, and to study the ways in which location-based messaging is used in home environments. Following this study we anticipate extending the capabilities of this system as suggested above.

\section{References}

1. O'Hara, K., Harper, R., Unger, A., Wilkes, J., Sharpe, B., Jansen, M.: TxtBoard: from textto-person to text-to-home. CHI '05 extended abstracts on Human factors in computing systems. ACM, Portland, OR, USA (2005)

2. O'Hara, K., Perry, M., Lewis, S.: Social coordination around a situated display appliance. Proceedings of the SIGCHI conference on Human factors in computing systems. ACM, Ft. Lauderdale, Florida, USA (2003)

3. Cheverst, K., Fitton, D., Dix, A.: Exploring the evolution of office door displays. In: O'Hara, K., Perry, M., Churchill, E., Russell, D. (eds.): Public, Community and Situated Displays: Social and Interactional Aspects of Shared Display Technologies. Kluwer, Dordrecht (2003)

4. Neustaedter, C., Brush, A.J.B.: "LINC-ing" the family: the participatory design of an inkable family calendar. Proceedings of the SIGCHI conference on Human Factors in computing systems. ACM, Montreal, Quebec, Can ada (2006)

5. Sellen, A., Harper, R., Eardley, R., Izadi, S., Regan, T., Taylor, A.S., Wood, K.R.: HomeNote: supporting situated messaging in the home. Proceedings of the 2006 20th anniversary conference on Computer supported cooperative work. ACM, Banff, Alberta, Canada (2006)

6. Sellen, A., Eardley, R., Izadi, S., Harper, R.: The whereabouts clock: early testing of a situated awareness device. CHI '06 extended abstracts on Human factors in computing systems. ACM, Montreal, Quebec, Canada (2006)

7. Beech, S., Geelhoed, E., Murphy, R., Parker, J., Sellen, A., Shaw, K.: The Lifestyles of Working Parents: Implications and Opportunities for New Technologies. HP Labs (2003)

8. UPnP Forum, http://www.upnp.org/standardizeddeps/mediaserver.asp 